Published in final edited form as:

Pediatr Cardiol. 2016 March ; 37(3): 512-518. doi:10.1007/s00246-015-1308-0.

\title{
Hospital Charges for Pediatric Heart Failure Related Hospitalizations from 2000 to 2009
}

Deipanjan Nandi ${ }^{1,}{ }^{*}$, Kimberly Y Lin ${ }^{1}$, Matthew J O'Connor ${ }^{1}$, Okan U Elci ${ }^{2}$, Jeffrey J Kim ${ }^{3}$, Jamie A Decker ${ }^{4}$, Jack F Price ${ }^{3}$, Farhan Zafar ${ }^{5}$, David LS Morales ${ }^{5}$, Susan W Denfield ${ }^{3}$, William J Dreyer ${ }^{3}$, John L Jefferies ${ }^{5}$, and Joseph W Rossano ${ }^{1}$

${ }^{1}$ Cardiac Center, The Children's Hospital of Philadelphia, Perelman School of Medicine at the University of Pennsylvania, Philadelphia, Pennsylvania

${ }^{2}$ The Children's Hospital of Philadelphia/Westat, Biostatistics \& Data Management, Philadelphia, Pennsylvania

${ }^{3}$ Department of Pediatrics, Lillie Frank Abercrombie Section of Pediatric Cardiology, Baylor College of Medicine, Texas Children's Hospital, Houston, Texas

4Johns Hopkins All Children's Heart Institute, Saint Petersburg, Florida

${ }^{5}$ The Heart Institute, Cincinnati Children's Hospital, Cincinnati, Ohio

\section{Abstract}

Background-Scarce data exists regarding costs of pediatric heart failure related hospitalizations (HFRH) or how costs have changed over time.

Hypothesis-Pediatric HFRH costs, due to advances in management, will have increased significantly over time.

Methods \& Results-A retrospective analysis of Healthcare Cost and Utilization Project Kids' Inpatient Database was performed on all pediatric HFRH. Inflation-adjusted charges are used as a proxy for cost. There were a total of 33,189 HFRH captured from 2000 to 2009. Median charges per HFRH rose from $\$ 35,079$ in 2000 to $\$ 72,087$ in 2009 ( $p<0.0001$ ). The greatest median charges were incurred in patients on extracorporeal membrane oxygenation $(\$ 442,134$ vs $\$ 53,998$ ) or ventricular assist devices $(\$ 462,647$ vs $\$ 55,151)$. Comorbidities, including sepsis $(\$ 207,511$ vs $\$ 48,995)$, renal failure $(\$ 180,624$ vs $\$ 52,812)$, stroke $(\$ 198,260$ vs $\$ 54,974)$ and respiratory failure ( $\$ 146,200$ vs $\$ 48,797)$, were associated with greater charges $(\mathrm{p}<0.0001)$. Comorbidities and use of mechanical support increased over time. After adjusting for these factors, later year remained associated with greater median charges per HFRH $(\mathrm{p}<0.0001)$.

Conclusions-From 2000 to 2009 there has been an almost two-fold increase in pediatric HFRH charges, after adjustment for inflation. Although comorbidities and use of mechanical support account for some of this increase, later year remained independently associated with

*Address for Correspondence, Deipanjan Nandi, MD, Cardiac Center, Children's Hospital of Philadelphia, $34^{\text {th }}$ Street \& Civic Center Boulevard, Philadelphia, PA 19104, Phone: 267-432-2674, nandid@email.chop.edu.

The authors have no conflicts of interest to declare. 
greater charges. Further study is needed to understand potential factors driving these higher costs over time and to identify more cost-effective therapies in this population.

\section{Keywords}

heart failure; cardiomyopathy; costs; value

\section{Introduction}

The management of both adult and pediatric heart failure is complex, with diverse etiologies and comorbidities. Patients who are hospitalized with pulmonary congestion but have adequate perfusion may only require a relatively brief hospitalization. Conversely, patients with poor perfusion and end-stage heart failure may require intubation, mechanical circulatory support, and other complex life-saving technologies. While the costs of such pediatric admissions are unknown, it is estimated that $\$ 30$ billion annually is spent on adult heart failure [1]. Although there is a lower incidence of heart failure in the pediatric population, given the complexity of care required for these patients, it is anticipated that the cost burden in pediatric HFRH may be similar to the adult population.

An important subset of pediatric HFRH are heart transplant admissions, which were found to have average hospital charges of greater than $\$ 450,000$ in 2006 , having increased by $\$ 170,000$ from 1997 [2]. A similar rise in charges is expected for the larger population of pediatric heart failure patients. With ongoing changes in the healthcare infrastructure, greater scrutiny on reimbursements and hospital charges for inpatient admissions will likely continue. To maximize both health care value for patients as well optimize the use of limited resources, costs first must be understood.

Thus, using inflation-adjusted charges as a proxy for cost, this study aimed to describe hospital costs for pediatric HFRH from 2000 to 2009 in the United States and assess factors associated with higher costs. We hypothesized that costs have increased over time and are associated with the increased use of mechanical support and greater incidence of comorbidities.

\section{Materials \& Methods}

\section{Data Source \& Study Population}

A retrospective review of the Kids Inpatient Database (KID) for the years 2000, 2003, 2006 and 2009 was performed. The KID is part of the Health Care Cost and Utilization Project, managed by the Agency for Healthcare Research and Quality, under the United States Department of Health and Human Services. The database was created to allow analyses of hospital utilization by children across the United States [3].

The KID consists of nationwide sampling of hospital admissions for pediatric patients. A systematic random sampling is used to select $80 \%$ of pediatric hospital admissions and complicated in-hospital births, and $10 \%$ of uncomplicated in-hospital births. To further ensure accurate representation of pediatric admissions, the discharges are sorted by state, hospital, diagnosis-related group (DRG), and a random number within each DRG. Hospitals 
included in the database are specialty hospitals, public hospitals and academic medical centers. The KID contains data from 2 to 3 million hospital discharges per year for children. There were 27 states that contributed data in the 2000 database, 36 states in the 2003 database, 38 states in the 2006 database, and 44 states in the 2009 database. The large sample size of the KID enables robust analysis of relatively rare diagnoses and procedures [4-7].

\section{Primary Outcome}

Serving as a surrogate for costs, the primary outcome was hospital charges per HFRH, which were obtained directly from HCUP KID. The KID is composed of more than 100 clinical and non-clinical variables for each hospital stay, including primary and subsequent diagnoses and procedures. All etiologies of heart failure were included, and these diagnoses were identified by International Classification of Diseases, Clinical Modification (ICD-9) codes. Charges were adjusted for inflation using data from the Bureau of Labor Statistics and are represented as 2009 US dollars [8].

\section{Demographic \& Clinical Data}

Additional data collected included admission and discharge status, patient demographics (e.g., sex, age, and race), length of stay (LOS), patient comorbidities, and hospital characteristics (e.g., location, size and teaching status). The hospital's location, teaching status, and size were determined by the American Hospital Association Annual Survey of Hospitals. Designation of a children's hospital was assigned based on information provided by the National Association of Children's Hospitals and Related Institutions (NACHRI). The primary payer was also identified.

\section{Statistical Analyses}

Descriptive statistics were presented as means (standard deviations) or medians (interquartile ranges) for continuous variables, as appropriate, and frequency counts and percentages for categorical variables. Chi-square tests were used to examine the change in comorbidities over time. Log transformation was performed to normalize the distribution of hospital charges per HFRH. Linear regression models were used to assess the factors associated with hospital charges. The unadjusted models included only the main effects of the factors. Adjusted models incorporated variables that were associated in univariable analyses, including calendar year, hospital characteristics, sex, age, congenital heart disease (CHD), arrhythmias, pulmonary hypertension, sepsis, acute cerebrovascular disease (CVD), respiratory failure, acute renal failure, and ventricular assist device (VAD), and extracorporeal membrane oxygenation (ECMO). Results from the univariable and multivariable regression models were reported as relative changes (i.e., anti-log of parameter estimates) with $95 \%$ confidence intervals and $p$ values. Since hospital charges were logtransformed, geometric means were used for analyses, although medians are reported here for ease of understanding. All variables had $<5 \%$ missing values, with the exception of race. The significance level for two-sided hypothesis testing was set at 0.05. Stata 12.1 (StataCorp, College Station, TX) was used to conduct the statistical analyses. 


\section{Results}

Descriptive characteristics of the pediatric HFRH included in this study are presented in Table 1. A total of 33,189 pediatric HFRH were captured over the entire study period. The underlying reasons for hospitalization were similarly distributed among the 4 years studied $(\mathrm{p}<0.001)$. Across the years studied, 58 to $62 \%$ of the hospitalizations occurred in patients with congenital lesions and 16 to $18 \%$ in patients with cardiomyopathy. Due to the nature of the available ICD-9 coding in the KID database, the heart failure etiologies for the remaining HFRH could not be determined. The mean age of admission across the years ranged from 5.3 to 5.9 years, although infants less than 1 year of age comprised the majority of cases for all the years studied. HFRH were evenly distributed by sex, with female patients representing $49 \%$ of all admissions. The mean length of hospital stay significantly increased from 2000 to 2009 (14.4 days [95\% CI 13.8-15.0 days] versus 17.1 days [95\% CI 16.5-17.7 days]; $\mathrm{p}<0.0001)$.

From 2000 to 2009, the median hospital charge per HFRH rose from \$35,079 (IQR, \$11,697 - \$91,357) to $\$ 72,087$ (IQR, $\$ 23,512$ - \$179,742). Use of mechanical support was associated with greater charge per HFRH for all years studied. Patients requiring ECMO had eight-fold higher median charges than those who did not ( $\$ 442,134$ vs $\$ 53,998 ; p<0.0001)$. Similarly, those requiring a VAD had eight-fold higher charges than those without a VAD $((\$ 462,647$ vs $\$ 55,151 ; p<0.0001)$. Higher charges per HFRH were also noted in those patients with severe comorbidities. Having had a congenital etiology of heart failure was associated with two-fold higher charges $(\$ 72,336)$ compared to other etiologies $(\$ 34,077$; $\mathrm{p}<0.0001)$. Renal failure, stroke, and respiratory failure were associated with three-fold higher charges, and sepsis was associated with four-fold higher charges ( $<<0.0001$; see Fig 1). Notably, the use of mechanical support and presence of severe comorbidities have all increased over time ( $\mathrm{p} \unlhd$.01; see Fig 2).

Charges per HFRH were greater in infants compared to older children (median charges $\$ 75,055$ vs $\$ 39,031 ; p<0.0001)$. Female gender was significantly but modestly associated with lower hospital charges than males (median charges $\$ 53,016$ versus $\$ 58,889 ; p<0.0001$ ). However, type of insurance was not associated with hospital charges $(\mathrm{p}=0.78)$.

Hospital characteristics such as being a teaching hospital or a children's hospital, but not hospital size, were significantly associated with greater median charges per HFRH (see Table 2). Patients that died during hospitalization incurred greater charges per HFRH than those who survived ( $\mathrm{p}<0.0001$; see Fig 1). Longer LOS was associated with greater charge. A LOS greater than 18 days (the highest quartile) was associated with median charges of $\$ 270,147$ while median charges for a LOS of less than 4 days were $\$ 10,633(p<0.0001)$.

In the multivariable analyses, all characteristics remained statistically significant after adjustment. ECMO was associated with a 2.3-fold increase in charges, VAD with 2.2-fold increase, and respiratory failure with 1.4-fold increase. LOS longer than 18 days was associated with 17-fold increased charges compared to a LOS less than 4 days. Inpatient stays at children's hospitals were associated with a 1.5-fold increase in charge. Charges in 2009 were 1.66-fold higher than charges in 2000 (see Table 2). 


\section{Discussion}

To our knowledge, this is the first study to explore how costs for pediatric HFRH have changed over time and to identify the primary factors contributing to overall costs. Using charges as a proxy for costs, the median hospital charge per HFRH doubled from $\$ 35,000$ to more than $\$ 70,000$ from 2000 to 2009 . We observed a 1.66-fold rise in inflation-adjusted charges per pediatric HFRH during the study period even after adjustment for mechanical support use, severe comorbidities, hospital characteristics and LOS. Taken together, these data indicate that there are unknown factors driving the increase in hospital charges per HFRH over the last decade beyond the higher costs associated with more medically complex patients and advancements in heart failure treatments.

The independent association between calendar year and charges per HFRH after accounting for patient complexity and increased mechanical support use was an unexpected finding. This finding persisted despite adjusting for a comprehensive list of potentially contributory patient and hospital characteristics. There may be several reasons for this observed increase in charges. First, there may be a true increase in resource utilization beyond the factors considered here. Indeed, recent data has shown that inpatient resource utilization has grown disproportionately higher for medically complex pediatric patients with other chronic diseases as well, including congenital heart disease [9]. Given the increasing prevalence of co-morbidities in pediatric heart failure patients observed in this study, there is likely increased utilization of resources and therapies associated with these conditions that were not able to be captured fully. Secondly, charges for hospital resources of all types may have increased. For example, individual hospital financial needs and changes in reimbursement may have led to higher hospital charges over time. It has also been speculated that hospital administrative data coding has improved with greater use of electronic health records, thereby allowing for more accurate, but increased, billing [10]. Further research is needed to explore these and other hypotheses that may potentially explain the observed increase in hospital charges over time. Future research into the rate of charge increase in other chronic pediatric conditions using this database may also be revealing.

We identified multiple factors that contributed to the higher charges per HFRH observed over time. The availability and utilization of advanced mechanical support, such as ECMO and VADs, in pediatric heart failure management has increased over time [11], as we redemonstrate in this study. These mechanical support devices incur substantial costs associated with the device itself as well as through the support staff, such as a perfusionist, required to operate the device during hospitalization. The high charges associated with ECMO and VAD, at a median charge of roughly $\$ 450,000$ per admission, likely contributes substantially to the overall financial burden of pediatric HFRH. This component of pediatric heart failure charges could potentially be modified to lower costs. In adults, a decrease in VAD-associated costs has been observed over time as facilities and healthcare providers have had increased experience with VADs, facilitating device implantation and use and reducing the risk of adverse events.

Furthermore, the use of VADs as destination therapy in adults, as opposed to its pediatric indication as bridge to transplant, may lead to a shorter length of stay, and presumably lower 
costs [12]. Whether a similar reduction in costs in the pediatric population will eventually occur over time remains to be seen. This could potentially be achieved by greater standardization of care across centers, regionalization of pediatric VAD centers, and greater use of VADs in outpatient settings.

Another important contributor of higher charges per HFRH over time was the increased prevalence of serious comorbidities (see Fig 2). With increased use of advanced mechanical support and improvements in heart failure management over time, patients are surviving longer, leading to an increased prevalence of comorbidities. It is well-documented that certain comorbid conditions, such as respiratory or renal failure, lead to higher costs [13]. In our study, we observed an increased prevalence of serious comorbidities over time, with two- to four-fold higher charges per HFRH associated with patients with respiratory failure, renal failure, sepsis, stroke, pulmonary hypertension, and arrhythmias (see Fig 1). Any future strategies to reduce or contain costs associated with inpatient pediatric heart failure management will need to consider approaches to rein in the additive costs incurred by more medically complex patients. Admissions captured in this study all have heart failure as an underlying diagnosis, but the database cannot distinguish if the primary reason for admission was secondary to exacerbation of a related co-morbidity, such as worsening renal function. Reducing costs requires the prevention of such admissions or mitigating associated LOS by ensuring ready access to not only advanced cardiac services, but to other highly skilled specialists, in both inpatient and outpatient settings.

In this study, we found higher charges for patients hospitalized in academic centers and children's hospitals. The increased availability of advanced mechanical and medical support at these centers likely contributes to these higher charges [14]. However, access to greater support may not be the only driver of higher charges in academic centers. Patients referred to academic center, often tertiary care centers, are known to have greater comorbidities and more complications, which in turn may lead to higher charges [13,15]. Additionally, prior studies have demonstrated that academic centers with traditional faculty/house staff models may deliver care at higher cost than centers with hospitalist systems, even when that care involves medically complex, subspecialty diseases [16-18]. Lastly, there is a concern that some children's hospitals may have regional de facto monopoly on access to advanced care such as mechanical circulatory support or heart transplant. While this may lead to more standardized care by groups with greater experience this can also translate to loss of regional competition when negotiating reimbursements and possibly lead to higher consumer costs, although the impact on charges would be difficult to predict [19].

LOS has previously been associated with higher cost in transplant patients and in patients requiring VADs [20,21]. Similarly, we show that longer LOS is associated with higher charges in all pediatric HFRH. We also found a significant increase in LOS over the study time period, suggesting that these patients are spending more time as inpatients. Increased LOS does not, however, fully account for the increased charge over time, as the average daily charge has also increased over time ( $\$ 2,436$ per day in $2000, \$ 4,215$ per day in 2009$)$. Patients may have longer LOS due to our increased ability to support them through more severe illness, or the use of mechanical support as a bridge to transplant. Thus, while LOS may be one of the drivers of costs, advocating for a shorter LOS for all patients may not be 
the answer. Rather, costs may be reduced by decreasing LOS for a subset of patients, minimizing ICU stays by developing intermediate care units, and continuing to develop outpatient pediatric VAD programs.

Of note, inpatient mortality was associated with greater charge per HFRH, but mortality did not change over the years studied, despite the increased medical complexity of patients receiving care in the later years. However, by the nature of this study, out-of-hospital mortality cannot be studied. Without out-of-hospital mortality, or any meaningful quality of life indicators, it is difficult to draw conclusions about the quality or value of the health care being delivered to pediatric patients with heart failure.

The majority of the pediatric HFRH were in patients less than one year of age, a group in whom higher charges were found. Indeed, being an older child was slightly protective and was associated with 0.91 -fold charges compared to infants. This may be secondary to hospital procedure regarding site of admission (neonatal ICU versus a regular ward), higher charges related to the needs of very young patients, or lower nursing-to-patient ratios for infants. Infants with heart failure have a high morbidity and mortality, so perhaps it is not surprising that this group would also incur greater charges [22]. Interestingly, female gender was associated with somewhat lower charges, 0.97 -fold charges per HFRH, compared to male gender. This is in contrast to a previous study that demonstrated higher charges for female pediatric heart transplant recipients [2]. Further, despite the statistical significance, the observed difference in charges may be of minimal financial or clinical significance. The reason and significance behind this finding is unclear and warrants further study.

There are several limitations that should be mentioned. As a retrospective analysis of an administrative database, we were limited by the existing data in the database. For example, clinical data to assess the severity of disease and comorbidities were not available. Also, as this database captures only inpatient charges and does not link visits associated with the same patient, we were unable to look at emergency department and clinic visits charges, or categorize costs associated with readmissions. Misclassification of the ICD-9 codes used to search the database may have occurred despite the rigorous quality metrics used by the database administrators[23]. However, any resulting misclassification would be nondifferential. Lastly, as with other cost-effectiveness studies, hospital charges were used as a proxy for actual costs, with costs being less than hospital charges [24,25,15].

\section{Conclusions}

From 2000 to 2009, there was a 1.66-fold increase in hospital charges per pediatric HFRH after adjustment for increased mechanical support use and prevalence of severe comorbidities. Further study is needed to characterize factors that are driving costs for pediatric HFRH and may be potentially modifiable. Understanding drivers of costs will help us devise strategies that can maximize healthcare value while optimizing the use of limited resources.

\section{Acknowledgements}

This work was supported by the National Institutes of Health research grant T32-HL007915 (to D.N.). 


\section{References}

1. Rosamond W, Flegal K, Furie K, Go A, Greenlund K, Haase N, Hailpern SM, Ho M, Howard V, Kissela B, Kittner S, Lloyd-Jones D, McDermott M, Meigs J, Moy C, Nichol G, O'Donnell C, Roger V, Sorlie P, Steinberger J, Thom T, Wilson M, Hong Y. Heart disease and stroke statistics--2008 update: a report from the American Heart Association Statistics Committee and Stroke Statistics Subcommittee. Circulation. 2008; 117(4):e25-146. doi:CIRCULATIONAHA. 107.187998 [pii] 10.1161/CIRCULATIONAHA.107.187998. [PubMed: 18086926]

2. Law SP, Kim JJ, Decker JA, Price JF, Cabrera AG, Graves DE, Morales DL, Heinle JS, Denfield SW, Dreyer WJ, Rossano JW. Hospital charges for pediatric heart transplant hospitalizations in the United States from 1997 to 2006. J Heart Lung Transplant. 2012; 31(5):485-491. doi:S1053-2498(11)01257-5 [pii] 10.1016/j.healun.2011.12.008. [PubMed: 22306440]

3. Healthcare Cost and Utilization Project (HCUP) Kids' Inpatient Database (KID). 2013

4. Day MD, Gauvreau K, Shulman S, Newburger JW. Characteristics of children hospitalized with infective endocarditis. Circulation. 2009; 119(6):865-870. doi:CIRCULATIONAHA.108.798751 [pii] 10.1161/CIRCULATIONAHA.108.798751. [PubMed: 19188508]

5. Koubeissi MZ, Syed TU, Syed I, Jordan J, Alshekhlee A, Kossoff EH. Hemispherectomy-associated complications from the Kids' Inpatient Database. Epilepsy Res. 2009; 87(1):47-53. doi:S0920-1211(09)00199-5 [pii] 10.1016/j.eplepsyres.2009.07.008. [PubMed: 19734011]

6. Shah RK, Patel A, Lander L, Choi SS. Management of foreign bodies obstructing the airway in children. Arch Otolaryngol Head Neck Surg. 2010; 136(4):373-379. doi:136/4/373 [pii] 10.1001/ archoto.2010.38. [PubMed: 20403854]

7. Stern RE, Yueh B, Lewis C, Norton S, Sie KC. Recent epidemiology of pediatric cochlear implantation in the United States: disparity among children of different ethnicity and socioeconomic status. Laryngoscope. 2005; 115(1):125-131. doi:00005537-200501000-00026 [pii] 10.1097/01.mlg.0000150698.61624.3c. [PubMed: 15630380]

8. Berndt, ER.; Cutler, DM.; Frank, RG.; Newhouse, JP.; Triplett, JE. Handbooks in economics. 1st. Vol. 17. New York: Elsevier,, Amsterdam; 2000. Handbook of health economics; p. $2 \mathrm{v}$

9. Berry JG, Hall M, Hall DE, Kuo DZ, Cohen E, Agrawal R, Mandl KD, Clifton H, Neff J. Inpatient growth and resource use in 28 children's hospitals: a longitudinal, multi-institutional study. JAMA Pediatr. 2013; 167(2):170-177. [PubMed: 23266509]

10. Neff JM, Clifton H, Park KJ, Goldenberg C, Popalisky J, Stout JW, Danielson BS. Identifying children with lifelong chronic conditions for care coordination by using hospital discharge data. Acad Pediatr. 2010; 10(6):417-423. [PubMed: 21075324]

11. Morales DL, Zafar F, Rossano JW, Salazar JD, Jefferies JL, Graves DE, Heinle JS, Fraser CD Jr. Use of ventricular assist devices in children across the United States: analysis of 7.5 million pediatric hospitalizations. Ann Thorac Surg. 2010; 90(4):1313-1318. discussion 1318-1319. doi:S0003-4975(10)01023-4 [pii] 10.1016/j.athoracsur.2010.04.107. [PubMed: 20868835]

12. Digiorgi PL, Reel MS, Thornton B, Burton E, Naka Y, Oz MC. Heart transplant and left ventricular assist device costs. J Heart Lung Transplant. 2005; 24(2):200-204. doi:S1053249803009744 [pii] 10.1016/j.healun.2003.11.397. [PubMed: 15701438]

13. Odetola FO, Gebremariam A, Davis MM. Comorbid illnesses among critically ill hospitalized children: Impact on hospital resource use and mortality, 1997-2006. Pediatr Crit Care Med. 2010; 11(4):457-463. [PubMed: 20595822]

14. Lowry AW, Morales DL, Graves DE, Knudson JD, Shamszad P, Mott AR, Cabrera AG, Rossano JW. Characterization of Extracorporeal Membrane Oxygenation for Pediatric Cardiac Arrest in the United States: Analysis of the Kids' Inpatient Database. Pediatr Cardiol. 2013

15. Wall TC, Fargason CA Jr, Johnson VA. Comparison of inpatient charges between academic and nonacademic services in a children's hospital. Pediatrics. 1997; 99(2):175-179. [PubMed: 9024442]

16. Auerbach AD, Wachter RM, Katz P, Showstack J, Baron RB, Goldman L. Implementation of a voluntary hospitalist service at a community teaching hospital: improved clinical efficiency and patient outcomes. Ann Intern Med. 2002; 137(11):859-865. [PubMed: 12458985] 
17. Bekmezian A, Chung PJ, Yazdani S. Staff-only pediatric hospitalist care of patients with medically complex subspecialty conditions in a major teaching hospital. Arch Pediatr Adolesc Med. 2008; 162(10):975-980. [PubMed: 18838651]

18. White HL, Glazier RH. Do hospitalist physicians improve the quality of inpatient care delivery? A systematic review of process, efficiency and outcome measures. BMC Med. 2011; 9:58. [PubMed: 21592322]

19. Department of Justice, Federal Trade Commission. Improving Health Care: A Dose of Competition. 2004.

20. Slaughter MS, Meyer AL, Birks EJ. Destination therapy with left ventricular assist devices: patient selection and outcomes. Curr Opin Cardiol. 2011; 26(3):232-236. [PubMed: 21430527]

21. Oostenbrink JB, Kok ET, Verheul RM. A comparative study of resource use and costs of renal, liver and heart transplantation. Transpl Int. 2005; 18(4):437-443. doi:TRI063 [pii] 10.1111/j. 1432-2277.2004.00063.x. [PubMed: 15773964]

22. Rossano JW, Kim JJ, Decker JA, Price JF, Zafar F, Graves DE, Morales DL, Heinle JS, Bozkurt B, Towbin JA, Denfield SW, Dreyer WJ, Jefferies JL. Prevalence, morbidity, and mortality of heart failure-related hospitalizations in children in the United States: a population-based study. J Card Fail. 2012; 18(6):459-470. [PubMed: 22633303]

23. US Department of Health and Human Services. [Accessed May 18, 2014] Guidelines for Ensuring the Quality of Information Disseminated to the Public. 2006. http://aspe.hhs.gov/infoquality/ guidelines/AHRQinfo.shtml. 2014

24. Fonseca AL, Schuster KM, Kaplan LJ, Maung AA, Lui FY, Davis KA. The use of magnetic resonance imaging in the diagnosis of suspected appendicitis in pregnancy: shortened length of stay without increase in hospital charges. JAMA Surg. 2014; 149(7):687-693. [PubMed: 24871698]

25. Del Prete SA, Ryan SP, Jacobson JS, Erichson RB, Weinstein PL, Grann VR. Safety and costs of treating neutropenic fever in an outpatient setting. Conn Med. 1999; 63(12):713-717. [PubMed: 10659471] 


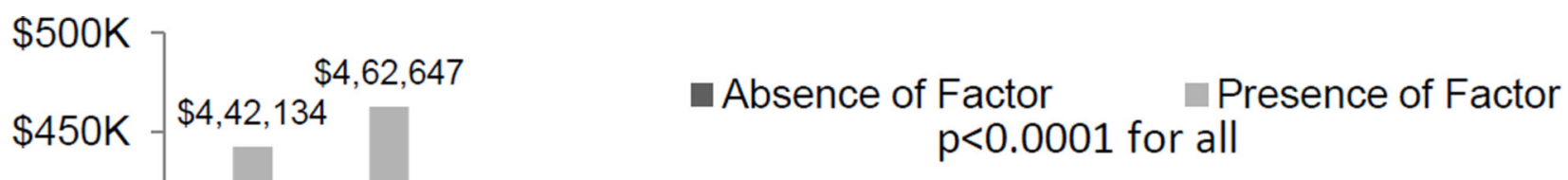

$\$ 2,07,511$

$\$ 200 K$

$\$ 150 K$

$\$ 100 \mathrm{~K}$

$\$ 50 \mathrm{~K}$

$\$ 55,151$
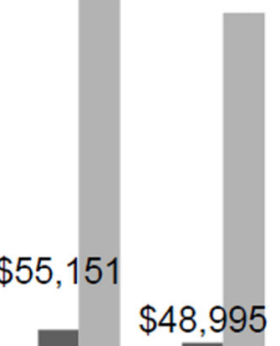

\section{$\$ 1,80,624$}

$\$ 1,98,260$

$\$ 1,46,200$

$\$ 1,47,941$

\$K<smiles>COCCOCCO</smiles>
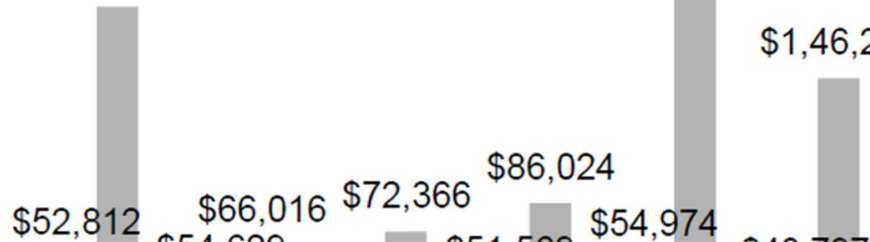

$\$ 66,016 \$ 72,366^{\$ 86,024}$

$\$ 54,974$

$\$ 75,055$

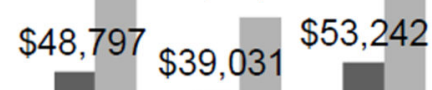

Fig. 1.

Comparison of median heart failure hospitalization charges by comorbidity/patient characteristic. Lighter bar represents presence of listed comorbidity, being an infant, or hospital mortality, which are all associated with higher charges. Extracorporeal membrane oxygenation (ECMO); Ventricular assist device (VAD); Acute renal failure (ARF);

Pulmonary hypertension (PH); Congenital heart disease (CHD); Arrhythmia (Rhythm); Cerebrovascular accident (CVA); Respiratory failure (Resp). Charges adjusted for inflation to 2009 US Dollars 


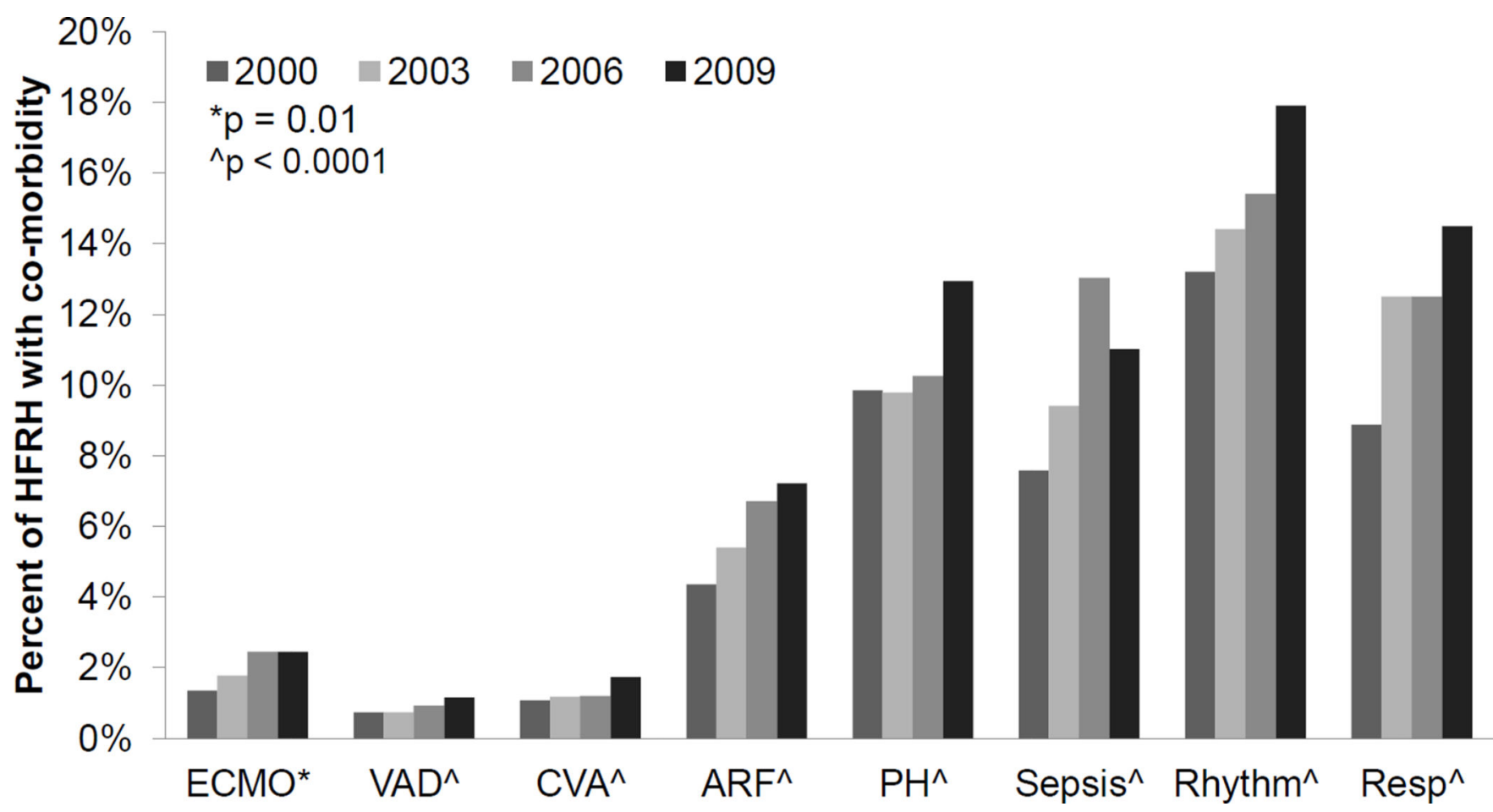

Fig 2.

Percentage of HFRH with stated comorbidities over time. Extracorporeal membrane oxygenation (ECMO); Ventricular assist device (VAD); Cerebrovascular accident (CVA); Acute renal failure (ARF); Pulmonary hypertension (PH); Arrhythmia (Rhythm); Respiratory Failure (Resp) 


\section{Table 1}

Patient Characteristics of Pediatric Heart Failure Hospitalizations

\begin{tabular}{lcccc}
\hline Variable $^{*}$ & $\mathbf{2 0 0 0}$ & $\mathbf{2 0 0 3}$ & $\mathbf{2 0 0 6}$ & $\mathbf{2 0 0 9}$ \\
\hline Admissions & 6,478 & 7,465 & 9,686 & 9,560 \\
Congenital disease & $3,803(58.7 \%)$ & $4,308(57.7 \%)$ & $5,940(61.3 \%)$ & $5,883(61.5 \%)$ \\
Cardiomyopathy & $1,124(17.4 \%)$ & $1,346(18.0 \%)$ & $1,566(16.2 \%)$ & $1,653(17.3 \%)$ \\
Charges, $\$$ \# & $\$ 35,079(\$ 11,697-$ & $\$ 49,987(\$ 16,184-$ & $\$ 63,461(\$ 20,551-$ & $\$ 72,087(\$ 23,512-$ \\
Hospital LOS, days & $\$ 91,357)$ & $\$ 120159)$ & $\$ 164,322)$ & $\$ 179,742)$ \\
Hospital mortality & $14.4(13.8-15.0)$ & $15.0(14.4-15.5)$ & $18.2(17.6-18.7)$ & $17.1(16.5-17.7)$ \\
Female & $468(7.2 \%)$ & $520(7.0 \%)$ & $670(6.9 \%)$ & $570(6.5 \%)$ \\
Age, years & $3,145(48.6 \%)$ & $3,724(49.9 \%)$ & $4,723(48.8 \%)$ & $4,719(49.4 \%)$ \\
$<1$ (Infant) & $5.3(5.1-5.5)$ & $5.9(5.7-6.1)$ & $5.3(5.2-5.5)$ & $5.5(5.4-5.7)$ \\
$1-12$ & $3,388(52.4 \%)$ & $3,672(49.3 \%)$ & $5,414(56.1 \%)$ & $5,118(53.7 \%)$ \\
$13-20$ & $1,551(24.0 \%)$ & $1,738(23.3 \%)$ & $1,808(18.7 \%)$ & $1,928(20.2 \%)$ \\
Non-white & $1,531(23.7 \%)$ & $2,034(27.3 \%)$ & $2,431(25.2 \%)$ & $2,476(26.0 \%)$ \\
Private insurance & $2,963(54.6 \%)$ & $2,890(51.4 \%)$ & $3,787(51.0 \%)$ & $4,400(53.3 \%)$ \\
\hline
\end{tabular}

Abbreviations: Length of Stay (LOS).

Data are presented as median (25th-75th percentile) for charges, mean (95\% confidence interval) for other continuous variables, count (\%) for categorical variables.

${ }^{\#}$ Charge data is adjusted for inflation to 2009 US Dollars 


\section{Table 2}

Relative change in hospital charges for pediatric heart failure related hospitalization for listed factors (multivariable model)

\begin{tabular}{crcc}
\hline & $\begin{array}{c}\text { Relative change } \\
\mathbf{( 9 5 \% ~ C I ) ~}\end{array}$ & p value \\
\hline \multirow{2}{*}{ Year } & 2003 vs 2000 & $1.26(1.22,1.29)$ & $<0.001$ \\
& 2006 vs 2000 & $1.46(1.42,1.50)$ & $<0.001$ \\
2009 vs 2000 & $1.66(1.62,1.71)$ & $<0.001$ \\
\hline Length of Stay (LOS) & LOS 4-7d vs 0-3d & $2.89(2.81,2.97)$ & $<0.001$ \\
& LOS 8-17d vs 0-3d & $6.06(5.90,6.22)$ & $<0.001$ \\
Hospital Characteristics & LOS >18d vs 0-3d & $16.9(16.4,17.4)$ & $<0.001$ \\
\hline Children's hospital & $1.47(1.43,1.50)$ & $<0.001$ \\
& Teaching hospital & $1.07(1.03,1.10)$ & $<0.001$ \\
\hline Patient Characteristic \& Comorbidity & ECMO & $2.28(2.13,2.43)$ & $<0.001$ \\
& Congenital heart disease & $1.22(1.19,1.25)$ & $<0.001$ \\
& Cerebrovascular accident & $1.21(1.13,1.30)$ & $<0.001$ \\
& Arrhythmia & $1.18(1.15,1.21)$ & $<0.001$ \\
& Acute renal failure & $1.29(1.25,1.35)$ & $<0.001$ \\
& Sepsis & $1.27(1.24,1.31)$ & $<0.001$ \\
& Fulmonary hypertension & $1.05(1.02,1.08)$ & 0.002 \\
& Age > 1 year & $0.91(0.89,0.93)$ & $<0.001$ \\
\hline
\end{tabular}

Abbreviations: Extracorporeal membrane oxygenation (ECMO) 